\title{
Heavy Quark diffusion from lattice QCD spectral functions
}

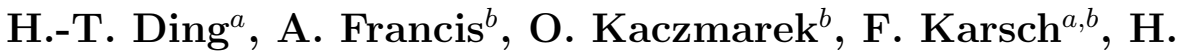 \\ Satz $^{b}$, W. Söldner ${ }^{c}$ \\ a Physics Department, Brookhaven National Laboratory, Upton, NY 11973, USA \\ b Fakultät für Physik, Universität Bielefeld, D-33615 Bielefeld, Germany \\ c Institut für Theoretische Physik, Universität Regensburg, D-93040 Regensburg, \\ Germany
}

E-mail: htding@bnl.gov

\begin{abstract}
We analyze the low frequency part of charmonium spectral functions on large lattices close to the continuum limit in the temperature region $1.5 \lesssim T / T_{c} \lesssim 3$ as well as for $T \simeq 0.75 T_{c}$. We present evidence for the existence of a transport peak above $T_{c}$ and its absence below $T_{c}$. The heavy quark diffusion constant is then estimated using the Kubo formula. As part of the calculation we also determine the temperature dependence of the signature for the charmonium bound state in the spectral function and discuss the fate of charmonium states in the hot medium.
\end{abstract}

\section{Introduction}

Experimentally a substantial elliptic follow of heavy quarks has been observed at RHIC [1. Various phenomenogical model studies suggest heavy quark diffusion coefficient $D \lesssim 1 / T$ to accomodate data (see e.g. Ref. [2]). Theoretically the heavy quark diffusion coefficient $D$ has been calculated by perturbative QCD in both leading and next-to-leading order as well as from AdS/CFT correspondence. At $\alpha_{s} \approx 0.2$, leading order $\mathrm{pQCD}$ calculation gives $2 \pi T D \approx 71.2[2$ ] while next-to-leading order calculation gives $2 \pi T D \approx 8.4[3$ ]. In the strong coupling limit $2 \pi T D=1$ is obtained from AdS/CFT correspondence [4]. Under such a circumstance a non-pertubative computation of heavy quark diffusion is neededt.

Through Kubo formula, the heavy quark diffusion constant $D$ relates to the vector spectral function as $D=\frac{\pi}{3 \chi_{00}} \lim _{\omega \rightarrow 0} \sum_{i=1}^{3} \frac{\sigma_{V}^{i v}(\omega, T)}{\omega}$, where $\chi_{00}$ is the quark number susceptibility and $\sigma_{V}^{i i}(\omega, T)$ is a mesonic spectral function in the vector channel. In the non-interacting case $\sigma_{V}^{i i}(\omega)$ has a $\omega \delta(\omega)$ term [6] and consequently gives an infinity diffusion constant, while in the interacting case $\omega \delta(\omega)$ will be smeared into a BreitWigner form [7] and leads to a finite diffusion. The fate of quarkonia states at finite

$\ddagger$ Here we focus on extracting diffusion coefficient from mesonic spectral functions, other ways can be found in Ref. [5]. 
temperature, which was suggested as a useful probe of QCD medium properties [8], can also be signaled by the deformation of spectral functions.

The mesonic spectral function is not directly accessible through lattice QCD simulations and can be obtained from the inversion of the following equation

$$
G_{H}(\tau, T)=\int_{0}^{\infty} \mathrm{d} \omega \sigma_{H}(\omega, T) K(\tau, T, \omega), \quad K(\tau, T, \omega)=\frac{\cosh \left(\omega\left(\tau-\frac{1}{2 T}\right)\right)}{\sinh \left(\frac{\omega}{2 T}\right)} .
$$

where the two-point correlation function $G_{H}(\tau, T)=\sum_{\vec{x}}\left\langle J_{H}(\tau, \vec{x}) J_{H}^{\dagger}(0, \overrightarrow{0})\right\rangle_{T}$ can be computed on the lattice. $J_{H}=\bar{q}(\tau, \vec{x}) \Gamma_{H} q(\tau, \vec{x})$ is a local mesonic operator and $\Gamma_{H}=\gamma_{i}, \gamma_{5}$ for vector $\left(V_{i i}\right)$ and pseudo-scalar $(P S)$ channels, respectively. The temperature $T$ is related to the Euclidean temporal extent $a N_{\tau}$ by $T=1 /\left(a N_{\tau}\right)$, where $a$ is the lattice spacing. We measured charmonium correlation functions on very fine $(a=0.01 \mathrm{fm})$ quenched lattices with a relatively large size of $128^{3} \times 96,128^{3} \times 48$, $128^{3} \times 32$ and $128^{3} \times 24$ at $0.73 T_{c}, 1.46 T_{c}, 2.20 T_{c}$ and $2.93 T_{c}$, respectively. The lattice parameters and part of the results have been reported in Ref. [9].

\section{Default model dependences of MEM results}

The Maximum Entropy Method (MEM) is used to extract spectral functions from correlators through Eq. (11) [10]. The input parameter of MEM is a default model (DM), which includes the knowledge about the spectral function, e.g. $\sigma(\omega) \geq 0$. The output spectral functions from MEM are reliable only if they show a small dependence on the input DMs. Thus it is very important to study the default model depedence of the output spectral functions.

From the left panel of Fig. 1 we see that the default model dependence of vector spectral functions at $0.73 T_{c}$ is very small. The location of the ground state peak is very close to the physical $J / \psi$ mass. The width of the peak is too wide to be interpreted as the physical width of $J / \psi$. We found no evidence for the existence of a transport peak at this temperature. In the right panel of Fig. 11 we show $\left(G(\tau T)-G_{r e c}(\tau T)\right)=$
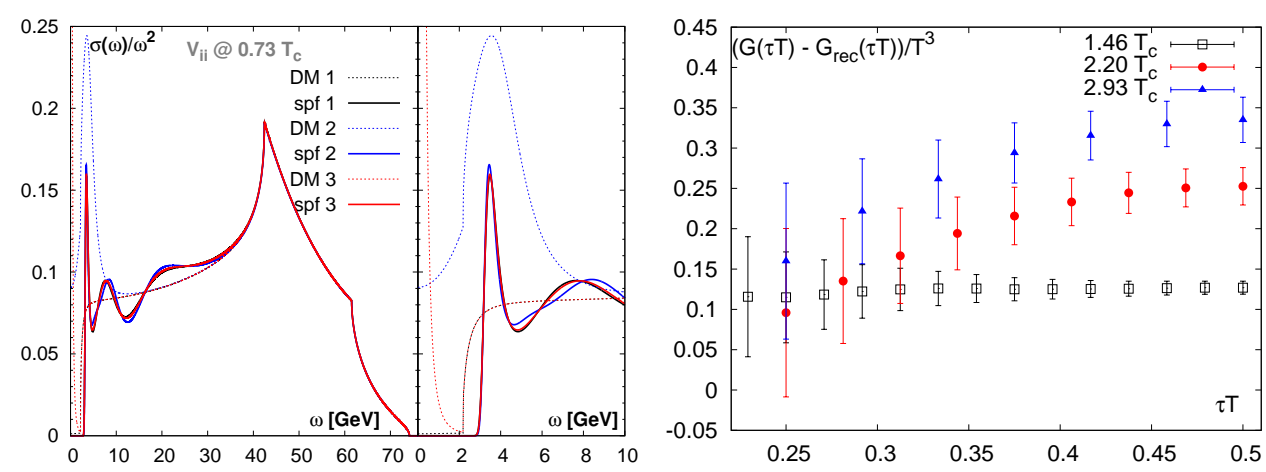

Figure 1. Left: Default model dependences of vector spectral functions at $0.73 T_{c}$. Right: differences of $G(\tau T)$ from $G_{r e c}(\tau T)$ as a function of $\tau T$. $G_{r e c}(\tau T)$ is the reconstructed correlation function from the spectral function at $0.73 T_{c}[9]$. 
$\int \mathrm{d} \omega K(\tau T)\left(\sigma(\omega, T)-\sigma\left(\omega, 0.73 T_{c}\right)\right)$ as a function of $\tau T$. The flatness of $G-G_{r e c}$ at $1.46 T_{c}$ indicates some small changes in the bound states and the rising feature with increasing distances at higher temperatures indicates $\left(\sigma(\omega, T)-\sigma\left(\omega, 0.73 T_{c}\right)\right)$ is negative in some low frequency region. To look into the detailed change of the spectral function from below to above $T_{c}$, it is crucial to investigate the spectral function itself.

In Fig. 2 we show the default model dependence of vector spectral functions at $T>T_{c}$. We first vary the low frequency (transport) part of the spectral function in the default model, where plots A1) and A2) show the low frequency and high frequency parts of the spectral function, respectively. A small default model dependence is observed. We then vary the resonance part of the spectral function in the default model, where plots B1) and B2) show the high frequency and low frequency parts of the spectral function, respectively. In particular, the first peak location in "DM 2" corresponds to the peak location of the spectral function at $0.73 T_{c}$. We observed that the ground state peak location in general shifts to higher energy region at $1.46 T_{c}$ and becomes flat at higher temperatures. Transport peaks have relatively strong default model dependences as seen in plot B2).

\section{Conclusion}

We summarize current uncertainties of transport peaks in the left panel of Fig. 4 and resulting heavy quark diffusion coefficients in the right panel. We found that $2 \pi T D$ at $1.46 T_{c}$ is close to unity and is slightly increasing with temperature. We also performed the default model dependence study in the PS channel and together with the uncertainties of vector spectral function are shown in Fig. 3, which suggests the dissociation of both $J / \psi$ and $\eta_{c}$ at $T \geqslant 1.46 T_{c}$.

\section{Acknowledgments}

This work has been supported in part by the Deutsche Forschungsgemeinschaft under

grant GRK 881 and by contract DE-AC02-98CH10886 with the U.S. Department of Energy. HTD thanks G. Moore for correspondences on NLO pQCD results of heavy quark diffusion.

\section{References}

[1] A. Adare et al. [PHENIX Collaboration], Phys. Rev. Lett. 98 (2007) 172301.

[2] G. D. Moore, D. Teaney, Phys. Rev. C71, 064904 (2005).

[3] S. Caron-Huot, G. D. Moore, Phys. Rev. Lett. 100 (2008) 052301.

[4] P. Kovtun, D. T. Son, A. O. Starinets, JHEP 0310 (2003) 064.

[5] S. Caron-Huot et al., JHEP 0904 (2009) 053, H. B. Meyer, New J. Phys. 13 (2011) 035008.

[6] F. Karsch et al., Phys. Rev. D68 (2003) 014504, G. Aarts et al., Nucl. Phys. B726 (2005) 93-108.

[7] P. Petreczky, D. Teaney, Phys. Rev. D73 (2006) 014508, G. Aarts et al., JHEP 0204, 053 (2002).

[8] T. Matsui and H. Satz, Phys. Lett. B 178, 416 (1986).

[9] H.-T. Ding et al., PoS LATTICE2010, 180 (2010), PoS LAT2009, 169 (2009).

[10] M. Asakawa, T. Hatsuda and Y. Nakahara, Prog. Part. Nucl. Phys. 46, 459 (2001). 

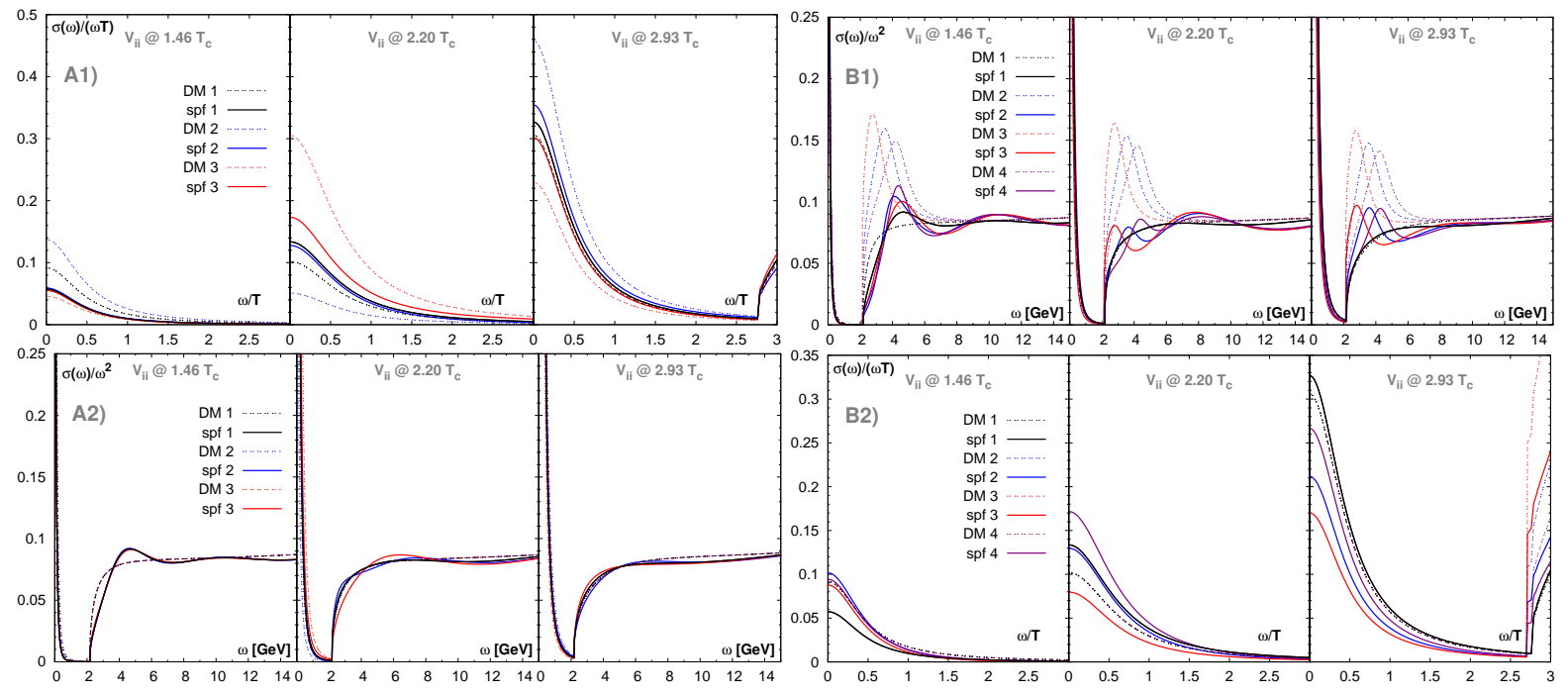

Figure 2. Default model dependences of vector spectral functions at $T>T_{c}$. The dotted and corresponding solid lines are for the different default models and output spectral functions, respectively.
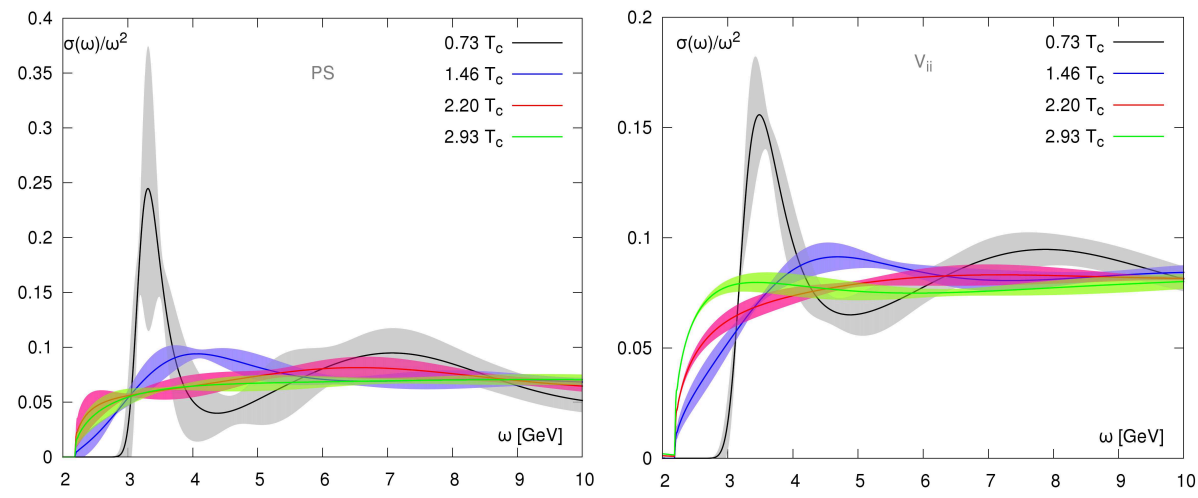

Figure 3. Uncertainties of output spectral functions in $P S$ (left) and $V_{i i}$ (right) channels at all available temperatures. The shaded areas are errors of output spectral functions from Jackknife and the solid lines inside the shaded areas are mean values of spectral functions.
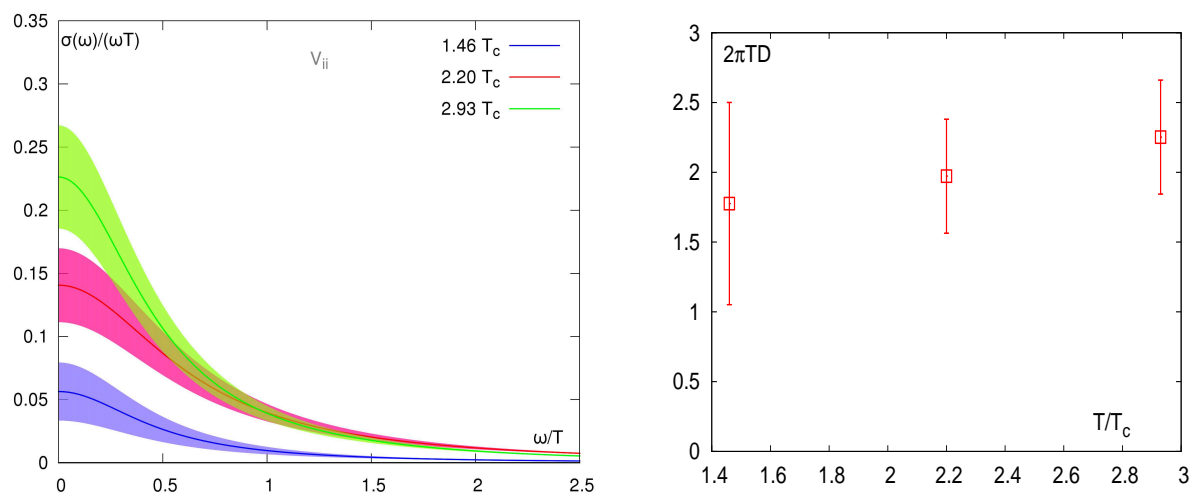

Figure 4. Transport peaks at $T>T_{c}$ and resulting diffusion coefficients. 